\title{
Formal modelling techniques in human-computer interaction *
}

\author{
G. de Haan ${ }^{a}$, G.C van der Veer ${ }^{\text {a,b }}$ and J.C. van Vliet ${ }^{a}$ \\ ${ }^{a}$ Free University, Amsterdam, The Netherlands \\ ${ }^{b}$ Twente University, Enschede, The Netherlands
}

This paper is a theoretical contribution, elaborating the concept of models as used in Cognitive Ergonomics. A number of formal modelling techniques in human-computer interaction will be reviewed and discussed. The analysis focusses on different related concepts of formal modelling techniques in human-computer interaction. The label 'model' is used in various ways to represent the knowledge users need to operate interactive computer systems, to represent user-relevant aspects in the design of interactive systems, and to refer to methods that generate evaluative and predictive statements about usability aspects of such systems. The reasons underlying the use of formal models will be discussed. A review is presented of the most important modelling approaches, which include External-Internal Task Mapping Analysis; Action Language; TaskAction Grammar; the Goals, Operators, Methods and Selection model; Command Language Grammar and Extended Task-Action Grammar. The prohlems associated with applying the present formal modelling techniques are reviewed, and possibilities to solve these problems are presented. Finally, we conclude with a discussion of the future work that needs to be done, i.e., the development of a general design approach for usable systems, and the need to focus attention on the practice of applying formal modelling techniques in design.

\section{Introduction}

The use of computers by non computer experts has sharply increased during the last decades. For this group of users, the computer is only a tool to get their work done, comparable to a pencil or a notebook. As such, to learn and use the computer system is an additional task derived from the application of this specific tool in the course of performing the primary task: the work being done (Van der Veer 1990), and therefore the effort of having to learn and use a computer system should be minimal. In Cognitive Ergonomics (the study of human-

* Requests for reprints should be sent to G.C. van der Veer, Dept. of Computer Science, Free University, De Boelelaan 1081a, 1081 HV Amsterdam, The Netherlands. 
computer interaction) the subject of study is the usability of computer systems to perform the user's tasks. From the viewpoint of the user, operating a computer system is not merely pressing buttons, but rather building an understanding of the system, as if it were a human partner (Oberquelle 1984). To analyse the computer system in this sense, a number of concepts have been introduced. Oberquelle (1984) has introduced the virtual machine, as the functionality of a system in terms of abstract (hence: virtual) functional units and their behaviour, without considering the details of the implementation and the hardware. In a similar way, the term user interface is used to include all perceptually and conceptually relevant elements and behaviour of a computer system that the user might know about, and should know to perform his tasks successfully. One might say that ideal or competent users, who have full knowledge of how to use a computer system to accomplish all possible tasks, know everything about the user interface in the Cognitive Ergonomic sense. In the remainder of our contribution we will use the term computer system with the same meaning as, and interchangeable with, the terms 'virtual machine' and user interface, to denote the system as far as it is relevant for a user, and hence may be either perceived directly, or may be conceptually conceived by interacting with the system.

In cognitive ergonomics, the usability of a system is assumed to depend on the organizational circumstances in which the computer is employed, on characteristics of the intended users of the computer system, on the tasks they have to perform, on the style of the dialogue with the computer system, and on the physical environment. Matters of concern are, among others, job and task analysis, task and computer experience, skill and problem solving, and measurements of office equipment. In Cognitive Ergonomics it is generally agreed upon that any system designed to be used by people should meet certain requirements. For example, Gould and Lewis (1985) state that a computer system should be:

- functional,

- easy to use,

- learnable,

- pleasant to use.

A computer system can be said to be usable to the extent that it meets these requirements. Usable systems, then, should provide the 
users with the functions they need to fullfil their tasks (functionality). The operation of the computer system should not require extensive mental or physical effort (easy to use). The operating procedures of the system should be easy to learn and easy to remember after periods of not using the system. And finally, using the computer system should be enjoyed by users. The joy of using computers will not be dealt with in this paper, because it is difficult, if at all possible, to approach this question with formal techniques.

It is necessary to distinguish the general concept of usability of computer systems in the sense of fulfilling the first three (or even all four) of the above-mentioned requirements, and the separate requirements. We will reserve 'usability' and 'usable', to refer to the quality of the system as a whole, and reserve 'easy to use' and 'ease of use', to refer to the narrow sense of demanding little mental or physical effort.

Formal modelling techniques or approaches can be used to represent the knowledge the user needs and/or the actions the user should perform to delegate his tasks to the computer system. We will not distinguish between the terms technique and approach and use these terms interchangeably. What is important is that applying formal modelling techniques results in models of knowledge and behaviour, which can be analyzed to investigate the extent computer systems fulfil the three requirements for usable systems.

\section{Models and levels of abstraction in human-computer interaction}

In order to develop a better understanding of what is involved in designing usable systems, it is necessary to take a closer look at the role of the user in operating a complicated device such as a computer, and introduce the notion of a user's mental model. Norman (1983) distinguished between three types of models: the user's mental model, the system image and the conceptual model of a computer system.

(a) The user's mental model is a model of the machine that users create or, according to Norman, which naturally evolves when learning and using a computer. This type of model does not have to be, and usually is not, accurate in technical terms. Instead, it may contain mis-conceptualizations, omissions and it does not have to be stable over time. However, a user's mental model is indispensable for the 
user to plan and execute interaction with the system, to predict, evaluate, and explain the behaviour of the computer, and to reduce the mental effort involved.

(b) The user's mental model is based on the system image, which includes all the elements of the computer system the user comes into contact with. As such, the system image includes all the aspects ranging from the physical outlook of the computer and connected devices to the style of interaction and the form and content of the information exchange. Although Norman (1983) excludes teaching materials and manuals from the definition of the system image, these could be included as well because they also shape the image of the system.

(c) The third type of model that Norman distinguishes is the conceptual model of the target system. This is the technically accurate model of the computer system created by designers, teachers and researchers for their specific purposes. As such, this type of model is an accurate, consistent and complete representation of the system, as far as user-relevant characteristics are involved.

The important point of Norman's distinction between three types of models is that in well-designed systems the conceptual model of the designers forms the basis for the system image, which in turn is the basis for the user's mental model. A good design starts with a conceptual model derived from an analysis of the intended users and the users' tasks. The conceptual model should result in a system image and training materials which are consistent with the conceptual model. This, in turn, should be designed in order to induce adequate users' mental models.

Formal modelling techniques in human-computer interaction are used to represent the knowledge users need about the operation of a proposed computer system, and to describe the actions users have to perform to delegate tasks in order to attain their task goals. By analyzing these representations, something can be said about whether the design of a computer system meets the required functionality, and to what extent the design will be easy to use and learnable. Formal representations show the complexity of the knowledge a user needs to acquire in relation to the tasks to be delegated.

Users need knowledge about a computer system for 'translation' in two directions. First, users come to the computer system with a set of 
tasks, and they will have to know how to translate and rephrase task delegation into the operating procedures and commands provided by the computer system. Secondly, after a command has been supplied to the system, the user must know how to interpret the behaviour of the system, and how to determine the success or failure of the task attempts. That is, users have to know how to translate their highly abstract task goals into the physical actions towards the computer system, and know how to relate the physical responses of the system to task goals and to task knowledge. In human-computer interaction it is common to distinguish several levels of abstraction in this specification/interpretation cycle (e.g., Moran 1981; Nielsen 1986; Frohlich and Luff 1989). Nielsen proposed the following levels of abstraction in the knowledge of computer users:

- Task Level,

- Goal Level, Semantic Level,

- Syntax Level,

- Lexical Level,

- Physical Level.

The essence of the notion of levels is that a user and a computer system communicate via certain types of languages that are different from 'natural' language. The correspondence between the meaning in the user's mind and the physical exchange of tokens between user and machine is far from trivial.

For example, a secretary who receives the instruction (task) to make a copy of a letter sent to a customer must know that copying a letter means to reproduce it on paper (goal level), which, in terms of a computer system, means to send a particular text file to a printer device (semantic level). It has to be known that commands must be submitted to the computer by specifying the operation (e.g. printing), a delimiter, an ordered list of arguments (e.g., printer destination first, letter identification second) and an end-of-command indicator (syntax level). Further, this user has to know that the letter is called 'smith.txt', that the command to send something to a printer is called 'print', and the name of the particular printer (lexical level). Finally, to submit the command the appropriate keys have to pressed (physical level).

Especially where it concerns larger and more complicated computer systems, it will be more difficult for a user to have a complete and 
flawless knowledge of each of these levels. Only expert users will have a mental model which will be consistent with the conceptual model of the system. In terms of Norman (1983), the user's mental model will usually differ from the conceptual model of the system. However, when a user interface is consistently structured and allows for a clear and straightforward mapping between the levels of abstraction then it will be easier for a user to develop an adequate mental model of the system. The key question is then: how to design user interfaces in such a way that the development of an adequate user's mental model is stimulated.

\section{Methods to enhance the usability of computer systems}

Once a system is designed and implemented, this topic may be approached relatively straightforward. When a computer system is successfully used, one may assume that the system did indeed stimulate the development of adequate mental models. For design purposes, empirical testing of the final product alone is not very practical; empirical testing is difficult and costly, it requires extensive effort, and its results come very late in the development cycle, often even too late to influence the design (Reisner 1983). A number of methods and tools has been developed to enable predictions regarding the end product's usability at an early stage of the design process.

One approach is to use design methodologies which involve active user cooperation during the whole process of design, to avoid that the design differs very much from what it was meant to be. Proposals for design methodologies like this frequently involve iterations to account for changing system requirements which may arise when users get familiar with using the new system.

Rapid prototyping can also be used to determine if a system, or parts of it, are usable. A prototype simulates the behaviour of the proposed machine more or less accurately (Diaper 1989).

Another approach is to use analogies and metaphors, which means that the design is built around an image borrowed from everyday life, like the furniture and tools in a clerk's office (e.g. the desk-top metaphor). Using a familiar image aims at letting users build on established knowledge, without having to start from scratch to build a mental representation of a system. 
A technique that designers expect to be really practical for improving the quality of design is to use guidelines and standards, or simply to look at the products of successful competitors. Applying guidelines like 'never use more than six colours' may save a large amount of time and effort otherwise spent on user testing (see for example Smith and Mosier 1984).

Finally, one may use modelling techniques to capture and analyze the knowledge the user needs to delegate tasks on a proposed computer system. Using these techniques, the analyst makes a more or less formal representation of the relations between the task-goals of the user and the operations needed to reach these goals. By analytic methods that estimate the complexity and consistency of the formal model, e.g. counting the number of rules or calculating the average number of parameters of each rule, something can be said about the functionality, ease of use and learnability of the proposed system.

Above, the various methods to ensure and improve the quality of designs have been presented separately. In actual design, however, combinations of methods need to be used, because no method can resolve all design questions, whereas combinations might produce better results. Which particular method to use depends on the specific question and the ability of a method to solve it, and on how much time and effort is needed to apply the method (Preece and Keller 1990).

In the following, we will concentrate on the use of formal modelling techniques to model user knowledge. We expect that formal modelling techniques have a number of advantages the other methods do not have, even though their use is not as well established as some other methods. The main advantage of formal models is the possibility to specify a design very precisely, without ambiguity. Using a formal notation also creates the possibility to automate parts of the implementation of user interfaces, and provides more possibilities for rapid prototyping and user testing. Moreover, analytic methods applied to formal models (aiming at establishing indexes of ease of use, learnability, and functionality) do not require a working system, as opposed to using empirical measurement techniques. Because only an initial specification of the user interface is required, formal modelling techniques may be applied very early in design to predict some of the usability aspects of the system. Also, since neither users nor working systems are involved, formal modelling can be applied at relatively low cost in comparison with techniques that require more than just a specification. 
We do not mean to say that empirical testing is not needed at all. Rather, we would like to suggest that applying formal models could enable the answer to certain questions in design earlier and with less effort than empirical testing, thereby leaving more resources for other methods to improve the quality of the design.

A further advantage, which formal techniques share with using analogies and metaphors, is that they closely adapt to Norman's notion of mental models. Formal models and metaphors both describe aspects of the conceptual model of the system and aspects of the user's mental model, but metaphors give an informal account of the conceptual model, and formal models represent aspects of it (e.g., the 'how-to-do-it' knowledge of a competent user) formally. Whereas metaphors refer to the conceptual meaningfulness of mental models, formal representations present the structure and consistency of an adequate mental model (i.e., a mental representation that is compatible with the conceptual model). The other advantages of formal models combined with their close connection with the notion of users' mental models makes them suitable candidates to serve as conceptual models to base design upon.

\section{An overview of formal modelling techniques}

In this overview, the most important or well-known of the formal modelling techniques to represent user knowledge will be treated. Applying formal models for user interface design includes some variant of a general procedure:

(a) the analyst makes a list containing what the users' goals can be and what users have to do in order to reach these goals;

(b) a model is built from the goal-operation sequences, using a more or less formal representation language, e.g., Backus-Naur Form;

(c) the model is restructured to represent the knowledge of a target group of users, such as 'novices', 'competent users', 'occasional users'. Many techniques model the knowledge of the 'ideal user', who is assumed to have full knowledge of operating the system and does not make any errors;

(d) the model is analyzed using some metric, e.g., providing indications of complexity of the transcription rule system, of the discriminabil- 
ity of sets of tasks or sets of objects, and of the consistency of representations at different levels of abstraction.

The differences between the various kinds of models include characteristics of the formal language (conceptual basis for representation), the levels of abstraction used in representing the communication between the user and the computer, and the methods used for the analysis of ease of use, learnability, and functionality. The most important reason behind these differences relates to the main goal the model is constructed for. Various categorizations of formal modelling approaches have been proposed (e.g. Green et al. 1988; Murray 1988; Oberquelle 1984; Rohr and Tauber 1984; Simon 1988; and Whitefield 1987).

The main problem in comparing these different categorizations of approaches to formal modelling is that they distinguish the modelling techniques to a large extent on scientific dimensions derived from the field of research, instead of practical considerations. For example, Green et al. (1988) ask whether a model describes performance or competence aspects of behaviour. Murray (1988) distinguishes prescriptive and descriptive models. In a similar vein, Simon (1988) uses the degree of idealization to distinguish between modelling ideal behaviour and real behaviour. Finally, Nielsen (1990) and Whitefield (1987) distinguish models, based on whether the model is owned or created by the user, the designer, the computer system, or the researcher, and what or who is being modelled.

Our intention is to analyze modelling approaches in relation to their merits for design from the point of view of usability. As systems are designed in order to enable task delegation by users to systems, we will base our categorization on this phenomenon. In delegating tasks to a computer four aspects are of importance.

(a) External tasks: Users have to perform tasks existing in a task domain outside the computer, which have to be rephrased in terms of the tasks that can be delegated to the computer.

(b) User knowledge: In delegating tasks, users need knowledge about the computer system, about the objects and operations the system knows about, and how to operate these in terms of physical actions.

(c) User performance: User performance is concerned with the users' behaviour in delegating tasks to the computer system. Users must perform certain actions, both mental, perceptual and physical. 
(d) The computer system: The system is the actual tool for task delegation, and, as a side effect, a main source for the user's knowledge of the interaction. It is the goal of the design process.

In accordance with these four aspects of task delegation, formal modelling techniques will be divided into four categories, each with its own specific purpose: task environment analysis, user knowledge analysis, user performance prediction, and representation for design purposes. The assignment of models to particular categories is not mutually exclusive. A formal representation can (and sometimes will) be used for different purposes, but assigning models to the category for which they were primarily developed will enable a fair judgement of their advantages as well as their restrictions.

\section{Models for task environment analysis}

With task environment analysis we apply a modelling technique that focusses on the characteristics of how to execute tasks in a certain task domain, and related knowledge of this task domain. The single example we show in this category is 'External-Internal Task Mapping' (ETIT, Moran 1983).

\section{ETIT}

Moran's External-Internal Task Mapping analysis is meant to analyze the relations between the external task domain (which refers to the tasks a user sets himself, or are set for a user, in relation to everyday reality) and the internal task domain (representing the delegation of suitable tasks to a computer system designed for application in the external task domain). Fig. 1 contains an example of text manipulation as a user's task, related to text manipulation using a simple editor on a computer (for a description of the editor, see Moran 1983).

The example shows the entities and the operations or tasks involved in the two contexts. In the external task space, several object types are referred to, such as characters, lines, and tasks are known, such as adding, moving, removing. The example editor, however, only knows a single object type 'string' as an entity that can be 'inserted', 'cut', or 'pasted'. In the analysis, several task-object combinations are excluded, because they do not make sense, such as an operation to split characters. Exempting these irrelevant operations, a number of map- 


\section{EXTERNAL TASK SPACE}

Terms: Character, Word, Sentence, Line, Paragraph (Text)

Tasks: Add, Remove, Transpose, Move, Copy, Split, Join

Excluded: Copy, Split and Join Characters

\section{INTERNAL TASK SPACE}

Terms: String

Tasks: Insert, Cut, Paste

\section{MAPPING RULES}

1. Split, Join Sentence $\rightarrow$ Change Text

2. Text $\rightarrow$ String

3. Add $\rightarrow$ Insert

4. Remove $\rightarrow$ Cut

5. Transpose $\rightarrow$ Move

6. Split $\rightarrow$ Insert

7. Join $\rightarrow$ Cut

8. Change Text $\rightarrow$ Cut String + Insert String

9. Move Text $\rightarrow$ Cut String + Paste String

10. Copy Text $\rightarrow$ Cut String + Paste String + Paste String

Fig. 1. External-Internal Task Mapping analysis.

ping rules can be determined which state how to translate a particular task from one environment to the other. The task 'copy a sentence' in the external world can be mapped on a task delegated to the editor, 'copy a string', which in its turn, must be rewritten as a combination of the actions 'cutting a string', 'inserting the string back in its original location', and 'inserting it elsewhere'. According to Moran, establishing the mapping between the objects and operations of the external and the internal tasks will make it possible to make inferences about the functionality, learnability and consistency of the user interface. ETIT should also be applicable in assessing the extent in which transfer of knowledge will occur between different user interfaces. Although ETIT is mentioned in the literature many times, we presently do not know whether ETIT has ever been applied to real systems.

Models to analyze user knowledge

The modelling techniques in this category employ a formal grammar to analyze and represent the knowledge the user needs to operate a user 
interface. This type of model may be used to compare the usability of different interfaces or different design options, and to predict differences in learnability.

More specifically, these techniques describe and analyze the knowledge the user must have in order to translate his tasks (originally represented by a user at the semantic or conceptual level) into the appropriate physical actions required to operate the system. We mention two modelling techniques in this category, Reisner's Action Language (Reisner 1981, 1983, 1984) and Task-Action Grammar (TAG, Payne 1984; Payne and Green 1986). Both techniques use a formal grammar to describe the task-action mappings, and both assess usability aspects by counting the number of rules, the depth of the derivation of rules and the number of exceptional rules. They differ with regard to the formal grammar they use.

\section{Action language}

Reisner's Action Language represents the task-action mappings in a notation called Backus-Naur Form (BNF), named after two of its authors (Backus et al. 1964). BNF is a formal notation to describe phrase structure grammars by means of a number of hierarchically organised rules. BNF is well known in computer science, where it is used, among other purposes, to describe what the legal or grammatically correct expressions are in programming languages like Algol and Pascal. In BNF, each rule specifies the relation between the more abstract term on the left-hand side and the more specific terms on the right-hand side by means of the 'is-defined-as' operator (::=). Alternatives are indicated by the 'or' symbol (I). In various extensions of the notation, succession may be indicated by the 'sequence' symbol $(+)$, and options are enclosed in brackets $([\ldots])$. BNF is a notation for context-free grammars which means that terms on the left-hand side are uniformly rewritten on the right-hand side independent of other terms and rules. As such it is not possible to indicate that, for instance, the form of a verb in English depends on whether the subject of the sentence is singular or plural.

In Cognitive Ergonomics BNF can be used to describe the legal sentences in the communication language the user has to use to delegate tasks to the computer system. In this way it models what a user has to know. Reisner (1983) extended BNF to include cognitive actions, written in angle brackets $(\langle\rangle)$, and physically observable 


\begin{tabular}{|c|c|}
\hline employ Dn & $\begin{aligned}::= & \langle\text { retrieve info. on Dn syntax }\rangle \\
& + \text { use Dn }\end{aligned}$ \\
\hline$\langle$ retrieve info. on Dn syntax $\rangle$ & $\begin{aligned}::= & \langle\text { retrieve from human memory }\rangle \\
& \mid\langle\text { retrieve from external source }\rangle\end{aligned}$ \\
\hline$\langle$ retrieve from human memory & $\begin{aligned}::= & \langle\text { RETRIEVE FROM LONG-TERM MEMORY }\rangle \\
& \mid\langle\text { RETRIEVE FROM SHORT-TERM MEMORY }\rangle \\
& \mid\langle\text { USE MUSCLE MEMORY }\rangle\end{aligned}$ \\
\hline retrieve from external source & $\begin{aligned} &::= \text { RETRIEVE FROM BOOK } \\
& \mid \text { ASK SOMEONE } \\
& \text { IXPERIMENT } \\
& \text { | USE ON-LINE HELP }\end{aligned}$ \\
\hline use Dn & $\begin{aligned}::= & \text { identify first line } \\
& + \text { enter Dn command } \\
& + \text { PRESS ENTER }\end{aligned}$ \\
\hline identify first line & $::=\quad \ldots$ \\
\hline enter Dn command & $::=$ TYPE D + type $n$ \\
\hline
\end{tabular}

Fig. 2. Action Language (Reisner 1983).

actions, written in capital characters. Fig. 2 shows a fragment of Reisner's Action Language or psychological BNF.

The first line in the example shows that the issuing of the command 'Dn' (to delete $n$ lines) consists of a cognitive action (to retrieve the correct syntax of the command), followed by a plain non-terminal (referring to how the syntax information is used and which keystrokes are involved). Although retrieving the needed information is a cognitive activity, and using it a as physical activity, both parts are rewritten in the same way. Reisner's action language has not been extensively used; Richards et al. (1986) used it to specify a graphical operating system shell (MINICON) with it. The only other application we know of stems from Reisner's own work on the pre-cognitive action language (Reisner 1981 ), in which two version of a drawing program are compared, one that does, and one that does not treat all the data objects in a uniform 
way. In this study, the non-uniform interface was characterized by the presence of additional rules to describe the exceptions, and as it was predicted, this interface turned out to be more difficult to learn and use than the interface which needed less rules to be completely described.

Reisner's work has indicated that BNF can be used to describe the knowledge the user needs to operate a computer system. However, in terms of the strength of expression, more powerful grammars can be, and are, used. Shneiderman (1982) introduced the idea of using a 'multi-party BNF' for representing the interaction decomposition regarding both 'partners' in human-computer interaction (see Innocent et al. (1988) for an elaboration of this concept). These formalisms, again, have not yet been elaborated for real-life situations. But BNF-like grammars of this type are still restricted to representation of sets of single rules.

A further development of BNF, Van Wijngaarden grammars (Van Wijngaarden et al. 1969), provides a formal representation technique for structured grammars that include the use of two levels of production rules. Payne and Greene (1983) show that set grammars (related to Van Wijngaarden's two-level grammars) enable the representation of 'family-resemblances' among rules. Only this new type of representation could account for the perception of consistency and inconsistency in syntax constructions, and, hence, could be used as a better model of a user's perception of an interaction language. This analysis led to the development of TAG.

$T A G$

Task-Action Grammar (Payne 1984; Payne and Green 1986) employs a more sophisticated semantic feature grammar than Reisner's BNF. 'Simple tasks' in TAG are represented by rules which can be rewritten in the same way as BNF rules, but in addition, TAG contains features which make it possible to describe tasks in terms of the meaning they may have for the user. In technical terms this means that it is possible to have rules describing the structure of sets of rules, which is not possible in the original versions of BNF. In terms of the user this means that tasks such as 'moving the cursor to the left' or 'moving the cursor to the right' are identical except for the indication of the direction. In fig. 3 cursor movement is used to illustrate TaskAction Grammar. The commands are listed in the 'Dictionary of simple tasks', from which a simple 'Rule schema' is derived that 
List of commands

move cursor one character forward

ctrl-C

move cursor one character backward

meta-C

move cursor one word forward

move cursor one word backward

ctrl-W

meta-W

List of features Possible Values

Direction forward, backward

Unit

character, word

$T A G$ definition

Dictionary of simple tasks

move cursor one character forward

\{Direction $=$ forward, Unit $=$ char $\}$

move cursor one character backward

$\{$ Direction $=$ backward, Unit $=$ char $\}$

move cursor one word forward

(Direction $=$ forward, Unit $=$ word $\}$

move cursor one word backward

$\{$ Direction $=$ backward, Unit $=$ word $\}$

\section{Rule Schemas}

Task [Direction, Unit] $\rightarrow$ symbol [Direction] + letter [Unit]

symbol [Direction $=$ forward] $\rightarrow$ 'ctrl'

symbol [Direction $=$ backward] $\rightarrow$ ' meta'

letter [Unit $=$ word] $\rightarrow$ ' $W$ '

letter [Unit $=$ character $] \rightarrow^{\text {' }} \mathrm{C}$ '

Fig. 3. Task-Action Grammar (Green et al. 1988).

illustrated the consistency of the syntax of the example. The user needs only knowledge of one general rule and of the 'features' Direction and Unit.

Green et al. (1988) have applied Task-Action Grammar to describe and explain the results of various experiments on command languages. An experiment is reported, in which subjects had to learn and use three applications, which were supplied with similar and different command languages. Learnability predictions were established for various formal modelling techniques, and for several design guidelines. A comparison between the predicted and the actual results showed TAG's predictions to be most accurate. Finally, Green and co-workers have applied TAG to describe several commercially available software packages, from 
which the general conclusion is drawn that extensions are needed when TAG is used for other purposes than the analysis of command language consistency.

\section{Models of user performance}

Methods for user performance predictions are the modelling techniques primarily targeted at analyzing, describing, and predicting user behaviour and time needed to get tasks done while using a particular computer system. Two often cited modelling approaches in this category are the GOMS model (Goals, Operators, Methods and Selection Rules) of Card et al. (1983) and the Cognitive Complexity Theory (CCT) of Kieras and Polson (1985).

Internally, the models used in this category are not very different from the models used to analyze the knowledge of the user, except for the fact that these models have a formal production-rule (if ... then ...) representation instead of a formal grammar. They do, however, differ with respect to the purpose of application. Whereas modelling techniques to analyze user knowledge describe and analyze what a user should or must know (without specifying how a user should apply this knowledge), the techniques to predict user performance describe and analyze what a user should know and, additionally, what a user should actually do in order to attain task goals.

As such, the GOMS and the CCT models are performance models, whereas the Action Language and the TAG representation are competence models. This difference may be illustrated for the case when the user may choose from alternative methods. In Reisner's Action Language and TAG the choice from alternatives is just described and specified by the 'or' (|) symbol, as a complete list of different possibilities, without indicating any conditions for actual choices. In GOMS and CCT, however, the goal is to predict user performance, and consequently the conditions for a user to choose an option must be specified in advance, e.g., by inferring individual users' strategies from observation.

The most serious implication from this is that GOMS and CCT require a complete specification of the task goal hierarchy of the user. Another consequence of this choice is, that GOMS and CCT implicitly claim that they can formally represent much more than Action Language and TAG claim, namely actual behaviour, instead of only 
GOAL: Edit-Manuscript

. GOAL: Edit-Unit-Task-until no more unit tasks

. GOAL: Acquire-Unit-Task

... Get-Next-Page-if at end of page

... Get-Next-Task

.. GOAL: Execute-Unit-Task

... GOAL: Locate-Line

.... [select: Use-String-Search-Method

$\ldots$ Use-Linefeed-Method]

... GOAL: Modify-Text

... . [select: Use-Delete-Word-1-Method

$\ldots$ Use-Delete-Word-2-Method

... $\quad$...

... Verify-Edit

Fig. 4. GOMS top level of an editing task (Card et al. 1983).

knowledge as a basis of behaviour. The task goal hierarchy which is needed for a GOMS or CCT analysis is in both cases a GOMS representation, or a hierarchical specification of the users' goals, operators, methods and selection rules.

\section{GOMS}

Fig. 4 presents an example of a GOMS representation of part of a text editing task.

As can be seen in the example, goals exist at several different levels of a task. A 'general goal' like editing a manuscript is initially subdivided into 'unit task goals', which correspond to the tasks the user knows how to perform. In general, 'unit tasks' correspond to the commands of a computer system. such as deleting a word, transposing two words, etc. in case of an editor. Unit task goals are further subdivided into a number of levels of 'subgoals', until they can be resolved by applying 'operators' or the 'elementary perceptual, motor, or cognitive acts', such as pressing a key, inspecting the screen or acquiring the next unit task. As a matter of fact, GOMS forms a family of models, because the level at which operators are defined may vary, and this level defines the granularity of a GOMS model.

Methods like using a string search or repeatedly pressing 'linefeed' to get the cursor in position are collections of operators. If there is more than one method to reach a goal, then selection rules determine which method will be used. For example, if the target position of the 
cursor is on the screen, the linefeed method is used, otherwise, the string search method is used. The time predictions GOMS generates depend on the level at which the operators are defined. In general, the predictions are based on the summation of the times needed to execute the elementary actions of the model, which include physical acts (pressing a key), perceptual acts (locating the cursor), and cognitive acts (making a selection).

A well-known member of the GOMS family of models is the Keystroke Level model. This model, however, lacks the analysis purpose of GOMS itself and is purely meant for predicting error-free, expert performance times. In the Keystroke Level model the user's tasks are analyzed at the level of unit tasks. The time to perform each unit task is estimated by adding the time to acquire the unit tasks, the time to execute the keystrokes in the associated commands, and the time needed for mental operators, which are inserted into the command sequences according to sets of heuristic rules. Fig. 5 shows an example of keystroke level analysis. General time parameters are estimated for different actions like 'press key or button', 'point with mouse', and 'mentally prepare'. The unit task illustrated requires the performance of a sequence of actions ("reach for mouse', 'point to word', 'select word' etc.), for which the corresponding time parameters are added to estimate the total execution time.

GOMS is probably the most cited formal model in human-computer interaction, even though GOMS is meant to be applied under rather restrictive conditions and for a rather limited purpose.

(a) As a performance model GOMS is restricted to predicting error free performance. In GOMS the cognitive load of a user is assessed by counting the number of active goals in memory. Lerch et al. (1989) used GOMS-based estimates of mental overload to predict error behaviour, which they showed to be valid for simple ('overload') errors, although GOMS still is unable to predict conceptual errors. This is a serious restriction. Roberts and Moran (1984) report that experts spend between 4 and 22 per cent of their time in correcting (only) serious errors (non-experts would struggle with errors even more often). Although Card et al. (1983) mention that it should be possible to apply GOMS to include error repair and non-expert performance, this has, to our knowledge, not been investigated in their studies. 


\begin{tabular}{|c|c|c|}
\hline Operator & Description and remarks & Time (sec) \\
\hline$\overline{\mathrm{K}}$ & $\begin{array}{l}\text { PRESS KEY OR BUTTON. } \\
\text { Pressing the SHIFT or CONTROL key counts as a } \\
\text { separate K operation. Time varies with the typing skill of } \\
\text { the user; the following shows the range of typical values: } \\
\text { Best typist ( } 135 \text { wpm) } \\
\text { Good typist ( } 90 \text { wpm) } \\
\text { Average skilled typist ( } 55 \text { wpm) } \\
\text { Average non-secretary typist }(40 \text { wpm) } \\
\text { Typing random letters } \\
\text { Typing complex codes } \\
\text { Worst typist (unfamiliar with keyboard) }\end{array}$ & $\begin{array}{l}0.08 \\
0.12 \\
0.20 \\
0.28 \\
0.50 \\
0.75 \\
1.20\end{array}$ \\
\hline $\mathbf{P}$ & $\begin{array}{l}\text { POINT WITH MOUSE TO TARGET ON A DISPLAY. } \\
\text { The time to point varies with distance and target size according } \\
\text { to Fitts's Law, ranging from } 0.8 \text { to } 1.5 \mathrm{sec} \text {, with } 1.1 \text { being an average. } \\
\text { This operator does not include the }(0.2 \mathrm{sec}) \text { button press that often } \\
\text { follows. Mouse pointing time is also a good estimate for other efficient } \\
\text { analogue pointing devices, such as joysticks. }\end{array}$ & 1.10 \\
\hline $\begin{array}{l}\mathbf{H} \\
\mathrm{D}\left(n_{D}, l_{D}\right)\end{array}$ & $\begin{array}{l}\text { HOME HAND(S) ON KEYBOARD ON OTHER DEVICE. } \\
\text { DRAW } n_{D} \text { STRAIGHT-LINE SEGMENTS OF TOTAL } \\
\text { LENGTH } l_{D} \mathrm{CM} \text {. } \\
\text { This is a very restricted operator; it assumed that drawing is } \\
\text { done with the mouse on a system that constrains all lines to } \\
\text { fall on a square } 0.56 \mathrm{~cm} \text { grid. Users vary in their drawing skill; } \\
\text { the time given is an average value. }\end{array}$ & 0.40 \\
\hline $\mathbf{M}$ & MENTALLY PREPARE. & 1.35 \\
\hline $\mathbf{R}(t)$ & $\begin{array}{l}\text { RESPONSE BY SYSTEM. } \\
\text { Different commands require different response times. The response } \\
\text { time is counted only if it causes the user to wait. }\end{array}$ & $t$ \\
\hline
\end{tabular}

Method for Task TI-BRAVO:

Reach for mouse $\quad \mathrm{H}$ [mouse]

Point to word $\quad$ P[word]

Select word K[YELLOW]

Home on keyboard H[keyboard]

Issue Replace command MK[R]

Type new 5-letter word 5K[word]

Terminate type-in MK[ESC]

$T_{\text {execure }}=2 t_{M}+8 t_{K}=2 t_{H}+t_{P}=6.2 \mathrm{sec}$.

Fig. 5. Keystroke model (Card et al. 1983).

(b) The model generates reasonably good predictions under rather specific conditions only. Card et al. (1983) base their predictions on GOMS analyses adapted to individual subjects, for instance, to 
account for differences in the criteria of selection rules. In the validation studies of the GOMS model, the subjects had to make changes to manuscripts from annotations, which is a rather limited task domain. Lerch et al. (1989) applied their GOMS analysis on a restricted set of tasks (financial calculations) for two commercially available spreadsheet systems.

(c) The GOMS analysis depends very much on the definition of unit tasks, but 'task the user knows how to perform' is not a precise definition, so that the analysist may have to rely on his own intuition in dividing the task into unit tasks (Wilson et al. 1988). In order to resolve this problem, Van der Veer (1990) defines unit tasks as 'elementary primary task that may not be decomposed into other primary tasks', where 'primary tasks', in turn, are defined as 'task the user wishes to perform, independent of the specific characteristics of the tools he will use'.

Although there are serious criticisms about how GOMS is applied to predict user behaviour, it is one of the most widely investigated models, and the value of GOMS as a heuristic method to gain insight in the users' tasks must not he underestimated. A GOMS representation is a very useful and systematic tool to describe the structure of decomposing the user's tasks in smaller elements. For structural description, a GOMS representation is much more useful than models of user knowledge or models for task environment analysis.

\section{CCT}

Another model to predict user performance is Cognitive Complexity Theory from Kieras and Polson (1985). CCT is primarily an implementation of the GOMS model in terms of an explicit production system; that is, Kieras (1988) has published a set of rules to rewrite the implicit if-goal then-action notation of a GOMS model into a real production system notation. Such a system is called a 'job-task analysis' which is regarded as a description of the process going on in the users' working memory. In this representation multiple conditions can be taken together by the AND operator, while the action part may consist of more than one action, including cognitive actions, such as the creation of (new) subgoals. An example of a fragment of a CCT analysis is shown in fig. 6, which represents a particular way to delete words, when using IBM's Displaywriter (Kieras and Polson 1985: pp. 374). The first 


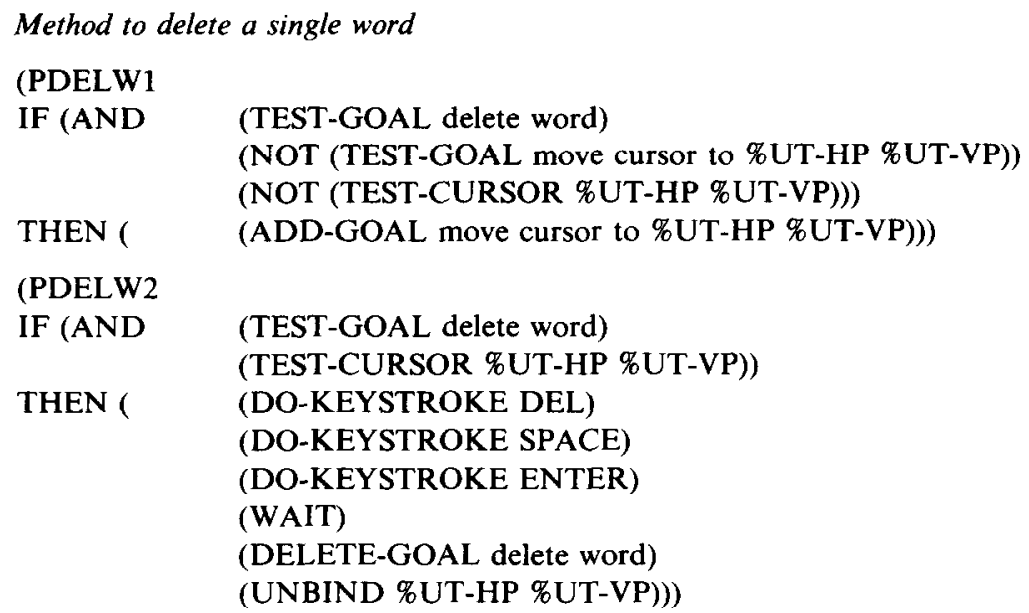

Fig. 6. Cognitive Complexity Theory (Kieras and Polson 1985).

production shows that the condition (goal is to delete a word, the goal position of cursor movement is not identical with the current position of the cursor) leads to the addition of the subgoal to move the cursor to the goal position. The second production shows that the condition (goal is to delete a word, cursor is at the goal position) leads to the sequence of actual actions that imply deletion and to the removal of the goal once this is fulfilled.

The aim of this representation is threefold:

(a) By estimating the time needed to execute productions, and, in particular, the operator parts of it, time predictions can be generated, based on the actual tasks and the task-job representation.

(b) Analyzing what the user has to do to operate a certain system in terms of production rules provides a uniform way to compare computer systems. When two computer systems have comparable functionality, the system that requires most production rules will be more difficult to learn and use. Kieras and PoIson have extended this point by stating that the ease of learning a new system will depend on the number of common rules between the new and the known system. Transfer of learning would only depend on the number of common rules, irrespective of, for example, confusion created by seeming commonality. 
(c) The production system representation can be used to analyze task-to-device mapping, representing the difference between the task-goal hierarchy of the user (how to do it) and the state transition of the system (how it works). Although, presumably no one will deny that performance is best when the user's expectations coincide with the behaviour of the system, Kieras and Polson have treated this subject too scant to say anything conclusive about it.

CCT may be used to compare systems in more or less the same way as the GOMS model and the models to analyze user knowledge or task environment. Because CCT uses the GOMS model, the same criticisms apply: the model has difficulty to cope with errors (Vossen et al. 1987), so that it may only be applied to routine expert tasks. According to Knowles (1988), CCT, by virtue of its reliance on GOMS is restricted in application to tasks involving no problem solving, besides that CCT relies on the quantitative aspects of representing knowledge at the expense of qualitative aspects. At present, CCT has been used mainly to analyze transfer of training effects both successfuly (Polson and Kieras 1985; Foltz et al. 1988; Polson 1988) and less successfuly (Vossen et al. 1987).

Critics state that CCT is not very clear about what actually constitutes a single production: 'Production rules can be rewritten in many different forms, thereby affecting the apparent complexity in terms of number of rules, number of times each one is used, etc.' (Green et al. 1988).

\section{Models of the user interface}

Models in this category are developed in relation to formal techniques for design specification. The models in this group aim at providing a complete and full representation of the 'virtual machine' (the computer system as seen from the point of view of a fully competent user). These models represent aspects of a computer system that are relevant for both the potential user and the designer, at the different levels of abstraction of human-computer interaction.

This category is exemplified by the Command Language Grammar (CLG; Moran 1981) and by Extended Task-Action Grammar (ETAG; Tauber 1988, 1990). Both are methods to describe the hierarchical structure of a user interface at the levels of abstraction mentioned 
before, in a related way. Apart from details such as naming, ETAG and CLG differ with respect to the formalism they use, and in some choices related to the organization and the main points of the representation.

\section{$C L G$}

Starting with a formal description of the tasks and the associated task-entities, and finally ending with the specification of the physical actions, the user interface can be precisely described for design purposcs in a top-down manner. Moran (1981) partitions the communication between man and machine into three components, each containing two levels. Each of the six levels is a complete description of the computer system at its level of abstraction:

Conceptual Component:

Communication Component:

Physical Component:
Task Level

Semantic Level

Syntactic Level

Interaction Level

Spatial Layout Level

Device Level.

Moran (1981) only discusses the first four levels and leaves the other two for future elaboration (and to 'classical' Ergonomics). The division into three components clearly indicates the major concern at each pair of levels (see Van der Veer 1989), but this is not strictly needed to understand CLG, and it will not be discussed here.

The division into six levels is directly related to considerations of good user interface design, based on the user's mental model. That is, the user comes to the computer system to get tasks done, and in order to do that, the tasks of the user have to be rephrased into the task language of the computer system and finally specified by physical actions of the user. The other way around, in order to understand the system, the user has to perceive the physical signals of the system, code them into meaningful symbol structures, and rephrase the responses of the system in terms of his primary tasks. Each of Moran's levels describes at a particular level of abstraction an aspect of this process, in terms of what has to be translated and how it is to be done. In this way, the output of each level is a further refinement of a previous level, or, in the opposite direction, an abstraction of the next level.

The purpose of the representation at the task level is to analyze the user's needs and to structure the task domain in such a way, that a 
computer system can play a part in it. The task level describes the structure of the tasks which can be delegated to the computer system. In order to use an interactive system, the user has to translate his tasks into operations the computer knows about.

The representation at the semantic level describes the set of objects, attributes, and operations, the system and the user can communicate about for the purpose of task delegation: for the system as data structures and procedures, and for the user as conceptual entities and operations on them.

The syntactic level describes which conceptual entities and operations may be referred to in a particular command context or system state, and how that is done, in terms of linguistic aspects (references to commands and objects, including the lexicon) and lexicographic aspects (the order of referencing, display areas). At this level it is specified, for instance, that there is a window to position delegation commands, and that the command to delete is 'delete' followed by the type of arguments to delete and a list of arguments.

Ultimately, the communication between man and computer is a matter of physical actions, such as sequences of key presses, movements of the mouse, meaningful signals like the 'beep' etc. The interaction level describes the translation of the reference names of commands and objects into the associated physical actions and the structure of the interaction, including typing rules and mouse manipulation conventions and the reactions and prompts from the system.

In Moran (1981), this is also the level where the treatment of CLG ends, but he adds that a full CLG analysis would also include a specification at the spatial layout level, and one at the device level. The former level describes the arrangements of the input and output devices, including display graphics, while the device level would describe all the remaining physical features. Fig. 7 presents a fragment of an electronic mail tool, described at the four highest levels of interaction. At the task level a description is shown of the task to read new messages (if any) and of the objects SEND-MESSAGE (which indicates a new message) and MESSAGE (which indicates any message, whether old or new). One of the constituents of the above mentioned task (check for new mail) is subsequently represented at semantic, syntax, and key-stroke level.

According to Moran (1981), three different points of view apply to CLG. 


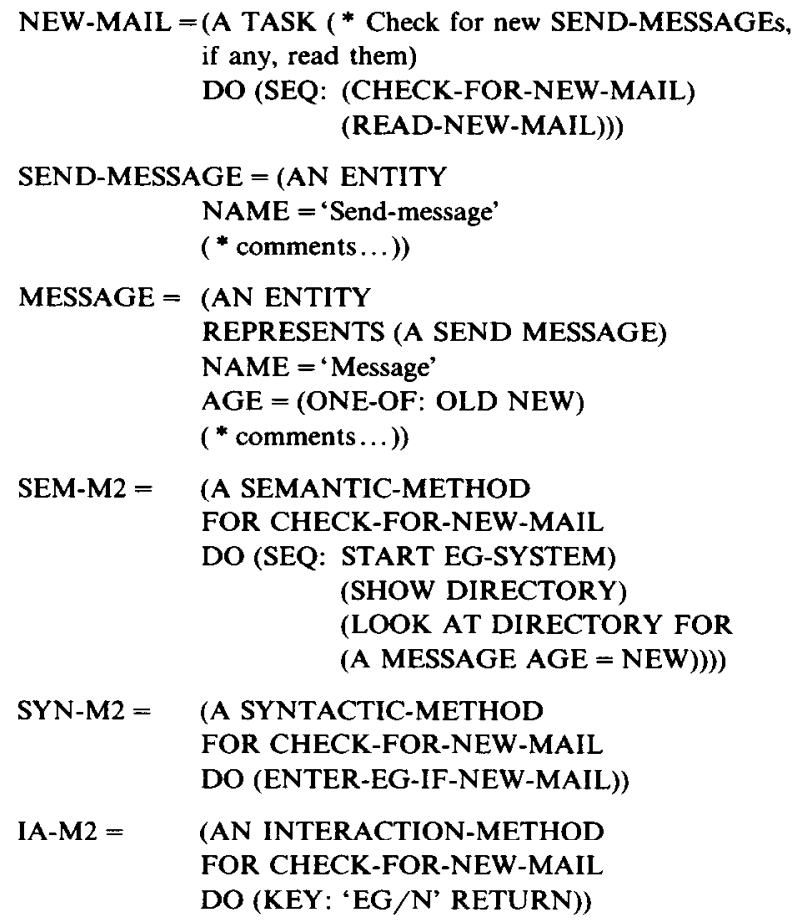

Fig. 7. Command Language Grammar (Moran 1981).

(a) The psychological view applies CLG as a model of an 'ideal' user's knowledge that shows the different kinds of knowledge that users have about systems. Moran, however, does not comment on the psychological validity of CLG as a model in this respect.

(b) The linguistic view uses CLG as a description of the structure of command language systems, which may be used to generate all possible 'command languages'. It should be noted that at the time of publication of CLG there was no uniform nomenclature of interaction styles (and, indeed, some currently well-known styles were not generally available), but CLG's claim is in principle valid for the description of all types of interaction mode.

(c) The design point of view applies CLG as a representation tool for specifying the system during the (top-down) design process to help the designer generate and evaluate alternative designs for the system. 
However, only the third view of CLG as a description of the conceptual model is really worked out, and most prevalent. To this might be added that there are more powerful, or less cumbersome, grammars to describe the linguistic structure of an interaction language. Furthermore, Moran leaves us with only a number of suggestions about how a CLG representation might be analyzed to predict or evaluate aspects of the system's usability, such as performance times, memory load and learning. Sharratt (1987) presents some results of using CLG as a specification tool in a practical design exercise, in which he asked students to use CLG to specify a design for a transport time-table system. Sharratt concludes that CLG is useful for design specifications, but that it carries many of the drawbacks of a strictly top-down design process and leaves little room for design iterations. Furthermore, CLG cannot be used to describe the relation between the tasks and the information on the screen. CLG, however, seems to provide a valid framework to model (competent) users's knowledge - at least from the point of view of the system designer (Van der Veer 1990).

\section{ETAG}

ETAG or Extended Task-Action Grammar (Tauber 1988, 1990) is in many ways comparable with Moran's CLG. Both are techniques to describe the human-computer interface from the point of view of the user (the 'virtual machine'), both employ the notion of levels of interaction, and both use formalisms to specify the contents of, and the mapping between these levels. Tauber (1988) used Task-Action Grammar (Payne 1984) to describe how users have to rephrase their tasks in terms of lower level rules, until arriving at the physical actions submitted to the computer system. Tauber prefers to use the concept of 'basic tasks' (different from Payne's 'simple tasks'), defined as 'tasks for which the system provides a single command or equivalent unit of delegation' (Tauber 1988). The system's basic tasks should be distinguished from the user's 'unit tasks', defined as 'elementary primary tasks that may not be decomposed into other primary tasks' (Van der Veer 1990).

Whereas TAG only provides levels for purely notational reasons, ETAG uses CLG's well-chosen levels of abstraction, adding some refinements. This is done, because ETAG is also aimed at formally specifying the 'user's virtual machine', including the task-related 
semantics of the computer system. The user's virtual machine (UVM) is defined by means of a canonical basis, an ontology borrowed from Psycho-linguistics (Jackendoff 1983). Basically, the ontological or

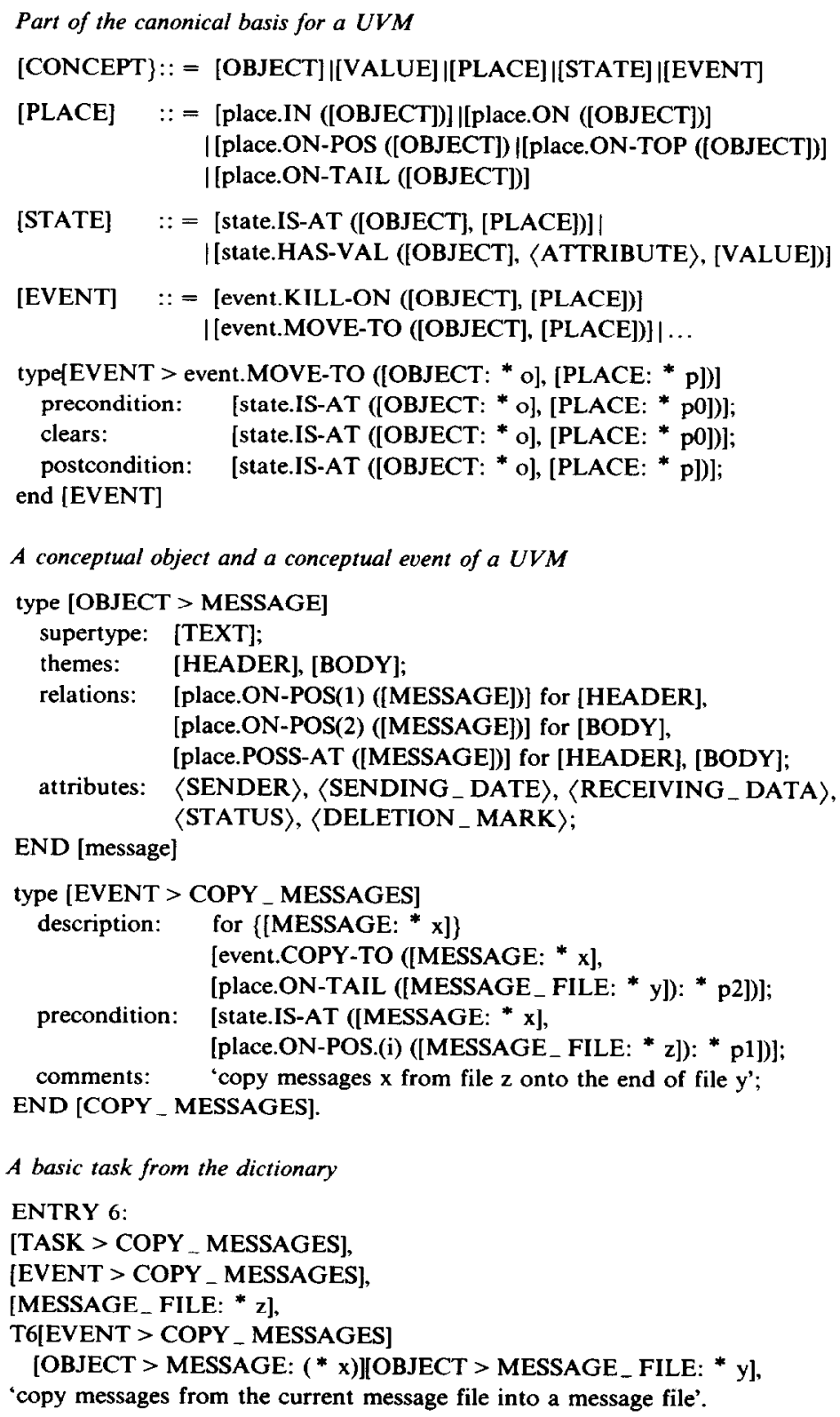

A basic task from the dictionary

ENTRY 6:

[TASK $>$ COPY MESSAGES],

[EVENT > COPY_ MESSAGES],

[MESSAGF_FILE: * z],

T6[EVENT > COPY, MESSAGES]

[OBJECT > MESSAGE: $(* x)][$ OBJECT > MESSAGE_ FILE: * y],

'copy messages from the current message file into a message file'.

Fig. 8. Extended Task-Action Grammar (Tauber 1990). 
canonical basis describes the world in terms of concepts (such as: objects, places, and states), attributes, relations between objects, functions of objects (such as: object being at places, e.g., on top of others), and events which change existence, functions, and relations (such as killing, moving and copying objects).

The canonical basis indicates relevance for the user, and should be part of the user's virtual machine. In terms of Norman (1983) the UVM is the conceptual model of the target machine and, as such, equivalent to a competent user's mental model. In terms of Kieras and Polson (1985) the UVM describes the 'how it works' knowledge the user needs. An example of a conceptual event in an electronic mail system is to 'mark for deletion', which sets the attribute 'deletion mark' for an object 'message' that resides at a place in 'message file'.

The next level in ETAG consists of the dictionary of the basic tasks. This level lists which basic tasks are possible, and how they relate to the concepts of the UVM. Fig. 8 gives an example of the higher levels of an interface specification in ETAG. Fragments of the UVM of an electronic mail system are illustrated including part of the 'canonical' basis (a concept hierarchy) and a description of an object (a message) and an event. An entry of the dictionary of basic tasks shows the formal description of the semantics of a basic task (copy a message).

The dictionary of basic tasks corresponds to the top level of the production rules, which use the feature grammar of Task-Action Grammar to describe how to perform the basic tasks in terms of still lower levels, until the commands for the computer system are fully specified. ETAG employs a refinement of the levels of CLG to structure the process of derivation by introducing levels to specify the syntax, the referencing style (e.g., pointing versus naming), the lexicon and the keystrokes, respectively.

ETAG, although originally designed as a modelling tool for user interface design, has already been applied for modelling user performance and user knowledge (Van der Veer et al. 1990).

\section{An evaluation of formal modelling techniques}

Formal modelling techniques as analyzed in the previous section, are representation methods to specify aspects of human-computer interaction. Models represent what a user has to know or to do in order to 
accomplish tasks by means of a computer system. Models can be used to analyze the similarities and differences in the way tasks are to be done with and without a computer system, to evaluate usability characteristics of human-computer interfaces, to predict certain aspects of user behaviour, and to formalize the hierarchical design of user interfaces in terms of the knowledge of the user at multiple levels of abstraction.

At this point, one could ask to what extent the present formal modelling techniques can be used successfully for these purposes. and if not completely successful, what the main problem areas are in using these techniques. To answer this question, it is necessary first, to determine the special requirements the modelling techniques should fulfil to specify user knowledge for the afore-mentioned purposes. We explicitly mention 'special' requirements, because we are concerned with formal models for specification purposes, namely, of user knowledge for the purpose of task-environment analysis, knowledge analysis, performance prediction, and representation for design.

In the past, others have suggested various requirements, some of which have been adapted, either completely or partially, and some have been rejected. For example, from Green et al. (1988) we accepted the requirement that formal models should be usable for designers. However, when they write that 'The model must contain a representation of the external semantics' (p. 38), they notice an important problem area of the present models, but not an overall requirement. Also, we do not think that it is necessary that 'The model must describe a reasonably complete psychology' (p. 38), as long as the resulting inferences are valid and useful for the desired purpose.

What we consider important is that formal models are tools to represent user knowledge for the purpose of computer system design. In the introduction, Norman's (1983) notion of models in human-computer interaction was discussed to stress the point that the design of a system should be based on a conceptual model, derived from an analysis of the intended users, and the users' tasks, in order to attain mutual consistency between the system image, user's mental models, training materials, and the conceptual model. From this basis, four requirements for representation techniques for design purposes can be put forward.

(a) A conceptual model should be based on both the point of view of the user, and provide a complete and accurate representation of the 
design. Hence, formal models should provide a complete description at the different levels of abstraction, of the intended system.

(b) Representation tools should have a wide applicability. In computer systems design, a modelling technique is of little use when one has to resort to another technique, for example, just when the style of interaction is to change from one to another. Therefore, formal modelling techniques should be applicable to a variety of different kinds of users, styles of interaction, and types of tasks.

(c) Formal models are used as conceptual representations of computer systems to analyze and predict usability aspects, about knowledge, and about performance. Inferences from analysis and prediction are useful to the extent that they are valid. The same applies to the modelling techniques on which the inferences are based.

(d) Just like computer systems are mere tools for their users, formal modelling techniques themselves are tools to perform the tasks of their own users: the designers. Therefore, within the broader context of design, formal modelling techniques themselves should fulfil the requirements of being functional, easy to use, and easy to learn and remember.

The requirements we have selected apply foremost to the modelling techniques for design specifications, but only because the design specifications are proposed with the most general intentions. CLG, with its three views, for example is intended for design specification, but also for user performance prediction and knowledge analysis. On the other hand, the requirements also apply to the techniques with more limited aspirations. For example, to analyze user knowledge, the representation should be a valid one, and include all the relevant aspects of the user's knowledge in a variety of circumstances and be usable for the analyst. One may imagine, however, that more specific purposes demand a different relative weighting of the requirements.

\section{The completeness of formal models}

The requirement of completeness means that a formal modelling technique should enable a complete specification of the user interface at all the levels of abstraction involved in using and in designing the interface. The modelling techniques reviewed in this paper fulfil this 
requirement to a smaller or larger extent, but none completely covers the whole interface. According to Green (1990) completeness is not a key requirement, and it may be better to have a number of limited theories, each of which covers its domain of application well, than to have a few large theories which cover more questions but each question only to a limited extent. As such, it may be possible to employ Cognitive Complexity Theory to predict performance times, Task Action Grammar to evaluate the consistency of an interface and Command Language Grammar to specify the interface for design. In the practice of design however, it is preferred to deal with only a limited number of methods since otherwise usability problems might arise. Regarding completeness, the main omissions in the present modelling techniques can be found at the highest and at the lowest levels of abstraction.

At the higher levels, an analysis of the user's concepts of the external tasks and those of the device are either omitted, mentioned without further specification, or the user's goal task hierarchy is much more rigidly specified than this will be the case in reality. For example, in Reisner's Action Language only attention is paid to the syntactic and lexical aspects of the users actions, and no attention is paid to the semantics of the interface. Payne and Green's TAG theory goes a step further, and list the semantic features of tasks, such as that the so-called 'clipboard' is involved in a cut and paste action. However, they do not in any sense describe the nature of clipboards as a temporary storage place for data. Moran's CLG would contain a description of the clipboard, but it would probably need a comment to fully explain its nature. At the moment only Tauber's ETAG is able to give formal account of these semantic features because it uses an ontology. Recent work by Payne (1987) also deals with the semantics of the interaction, especially those of the device being used.

At the lower levels of abstraction generally a description is missing of the visual presentation of the interface, especially in relation with the state of the system. Regarding the TAG theory, Green et al. (1988) write that: 'it [TAG] does not exhibit the relation between actions and system display; as far as TAG is concerned, the VDU screen could have been turned off' (p. 30). Although Moran (1981) explicitly mentions a spatial layout level, it has not been specified any further, and therefore none of the present modelling approaches is able to address the presentation component. 


\section{The width of applicability of formal modelling techniques}

The requirement of the width of applicability means that a formal modelling technique should be applicable to a variety of user-populations, types of tasks and ways of interacting with a computer. Here also, coverage is limited.

In the first place the knowledge or the performance of the user that is typically described refers either to the ideal user or to the competent user. The ideal user is taken to be one who has perfect knowledge and is only engaged in error-free and most efficient performance. On the other hand, the competent user is only perfectly knowing, but may commit performance errors. Even if the focus is on evaluating the interface as a whole, it is difficult to apply findings to real users, and especially to novice users, who may be characterized by their imperfect and even erroneous knowledge of the system (e.g., Briggs 1990). This point is not really problematic, because it refers only to the inability to predict task performance by individuals, whereas we are more concerned about evaluation of design and prediction of task performance in general. As an exception, CCT has been applied to model performance of non-expert users.

Secondly, except for Moran's ETIT analysis all the models essentially employ a context-free grammar or an equivalent method of representation, which means that performance on a given task is viewed independent of any other tasks and that only isolated tasks can be represented. The assumption that task performance is independent of for instance previous tasks does not seem to be in accordance with the reality of computer use.

The last point is the consequence of both the use of a context-free grammar, and the lack of a specification of the visual presentation component. Presently, it is not possible to apply the modelling techniques successfully to model other tasks than those requiring little or no control or revision of planning by the user during execution. In reality however, users do control their tasks based on the knowledge of delegation of other tasks (both in parallel and in sequence), and on the perception and interpretation of information on the screen and other system responses. Rassmussen (1987) points out that because of this restriction, formal models may eventually only apply to the least important and least interesting bits in human-computer interaction. Additional or alternative modelling techniques are required to address 
the dynamics in user task performance, like the PUMS approach (programmable user models) which covers problem solving to some extent (Young et al. 1989), or the Action Facilitation approach which is focussed on facilitating and inhibiting factors for task performance (Arnold and Roe 1987; Roe 1988).

\section{The validity of formal models and modelling techniques}

The third requirement of formal modelling techniques concerns the validity of the analyses and predictions delivered. This requirement does not only apply to task environment, knowledge analysis, and performance prediction. The representations for design purposes also carry a notion of what constitutes a good design, by the implicit choice to include certain features in the model as relevant and leave others out. Here, few problems can be mentioned; within the limited field of application for which the formal modelling techniques have been proposed, it has been shown that they indeed do what they are supposed to. To name a few, Reisner (1981) has shown that action language can be used to predict differences in ease of use between user interfaces. Card et al. (1983) and Polson (1987) mention a number of experiments in which user performance was predicted reasonably well by their respective models. The experiments described by Lerch at al. (1989) show experimental application of formal modelling to knowledge of commercially available systems, where both execution times and certain types of errors were succesfully predicted. Finally, Payne and Green (1989) describe an experiment in which Task-Action Grammar was successfully used to address subtle differences in the usability of interfaces, that other modelling techniques could not address.

There are several limitations connected to the afore-mentioned and other validation studies. The validation studies have generally used very simple user interfaces, they have almost always been performed by or under the supervision of the original authors of the method, and the studies have hardly ever taken place outside the research laboratory using full blown interfaces. Apart from establishing the utility of formal models by others than experts of the particular modelling technique, there is a need to establish the validity of the modelling techniques when used by non-experts, and a need to seek the limits of their applicability in real design. 


\section{The usability of formal modelling techniques}

A final requirement formal models have to satisfy is being of use in the practice of interface design. Here, the relevant points are to what extent formal models can be used in all stages of design, the adaptation to other techniques used by designers, and the usability aspects of applying formal models.

As was argued before the present approaches to formal modelling do not cover all the aspects and levels of abstraction of user interfaces. The current techniques cannot completely specify the semantics of the computer system, the presentation component and other reactions of the system. Presumably, the most important factor in this is the lack of a specification of the semantics of the system, including the "how it works' knowledge. because when the conceptual model of a design is known then it may be easily used, e.g., to guide the choice for a particular screen layout or for the contents of an error message. At present we can only say that work needs to be done in this area.

A second point is that formal modelling techniques are usable in design to the extent that they can be integrated with other techniques used by designers. As such, the use of formal models should both adapt to the very first stage of interface design, namely task analysis, and adapt to the final stages of design, such as software engineering, prototyping, and testing. In other words, formal models are tools to communicate very precisely about designs, but in rather abstract terms, which in some way or another have to be related to the real world. Formal modelling techniques may be expanded and adapted to the complementary methods that are applied in design. Summersgill and Browne (1989) report an attempt to integrate what they call 'functionality centred' and 'user centred' design techniques. Walsh (1989) notes that, although there is a gap between the techniques and notations of task-analysis, formal modelling, and software engineering. this is only a matter of a different focus, and not a matter of the inability to understand each other's language. Regarding the relation between formal modelling and task-analysis, what needs to be done is to find a way to translate the informal or semiformal representations of the tasks of users into a formal representation of the conceptual objects and operations which should be used in delegating tasks to the computer system. The relation between modelling user knowledge and software engineering is probably even less problematic, because both already use 
formal models. These models do, however, differ with respect to exactly what is modelled: aspects of the user or aspects of the interaction. Barnard and Harrison (1989) propose an interaction framework to model user-system behaviour as a bridge between modelling user knowledge and modelling system behaviour. Another promising development is the object-oriented approach, which may make it possible to create bridges between both task analysis and user knowledge modelling, and software engineering. Eventually, it may be possible to use the very same objects to represent the tasks and task entities of the users' task world, the conceptual objects and operations of the user interface, and the data objects and procedures of the computer system.

A third point concerns the usability of formal models for designers. The present formal modelling techniques are not easy to use and demand substantial effort. For example, Wilson et al. (1988) report that applying formal models does often require a high level of expertise on behalf of the designer. According to Sharratt (1987), who studied Command Language Grammar, this is especially the case when formal design specifications are changed, or design alternatives are to be compared. To attack this problem, there is a need for something like a designer's workbench, built around a particular formal modelling technique, or for providing facilities to employ different formal techniques. The most important thing the workbench should provide is a design approach to guide and structure design decisions. Furthermore, several tools will be necessary to relieve the designer from much of the administrative work, such as generating and changing formal design models (e.g., specialized editors that enable automatic semantic consistency checks, and templates for formalizing design attempts). Together, the design approach and the tool set should facilitate the integration between task analysis, formal modelling and implementation. The workbench should also provide for facilities to decrease expertise required in dealing with the formalism as such, by providing adequate on line help and explanation facilities during the different stages of design (see Van der Veer et al. (1990) for an example of a prototype of this type of tools).

\section{Concluding remarks}

In this paper it was argued that people use computers as mere tools to perform the tasks they have to do, and therefore the computer 
system should provide for the functions to enable users to delegate their tasks, and minimal effort should be required to learn and use the system. Furthermore, computer users create mental models of the system they are working with, which help then to explain the behaviour of the computer system, and serve to aid in planning their actions, thus reducing the mental effort required. It was argued that the designer of a computer system should consider the development of users' mental models, by taking a conceptual model as the basis for the design. Conceptual models can provide an accurate, consistent, and complete specification of the design, at different levels of abstraction in the knowledge of computer users. There are various, partially complementary methods to enhance a system's usability, each with specific advantages and disadvantages.

This paper focussed on formal modelling techniques to represent the knowledge users need to operate computer systems, which have the advantages of formality, early applicability, relative time and cost inexpensiveness, and usability as conceptual models. Various types of formal models were reviewed, according to their primary purpose: models for task environment analysis, models to analyze user knowledge, models to predict user performance, and representation models for design purposes. Formal modelling techniques should meet four special requirements, in addition to the general requirements imposed on formal systems and specification tools. For each of these requirements (completeness, wide applicability, validity and usability) the problems encountered with the present formal modelling techniques were discussed, along with possible solutions.

Regarding the question of what has to be done from now on, we can make a threefold distinction between addressing the limitations of the current models, the ongoing development of user-oriented computer systems design. and the use of formal modelling techniques in the design practice.

(a) The limitations of the present modelling techniques have to be addressed. Regarding the requirement of completeness, the present modelling techniques have to be extended, or alternative techniques need to he developed to enable (1) the specification of the visual presentation component, and (2) the specification of the semantics of the tasks and devices, in addition to task-action mappings. Where it concerns the requirement of a wide applicability, stronger 
modelling techniques have to be developed to address (1) the context sensitive aspects of the user-computer interaction, such as multi-tasking, and (2) the dynamic aspects of task control and planning, and the presentation of information and other reactions from the system. The requirement of validity demands validation studies to be done by independent researchers, using real computer systems outside the laboratory. Regarding the usability of formal models, opportunities should be investigated to bring about an integration between the techniques for task analysis, formal modelling techniques and software engineering methods. Also, tools should be developed to reduce the additional amount of effort and expertise, imposed by the present formal modelling techniques.

(b) Several remarks have been made about how to improve aspects of design approaches to increase the usability of computer systems. The question is now, how to develop a better design approach, which includes all improvements needed. On the basis of the previous discussion, it may be clear that we are convinced that a user-oriented design approach should be based on a conceptual model: an accurate, consistent and complete specification of the intended system, at the different levels of abstraction, and, most importantly, considering the knowledge of the intended user. A formal modelling technique can be chosen, either from this overview or from another source, on the basis of the four requirements previously discussed, and possibly additional requirements, such as opportunities to extend the model, or to integrate it with current design tools. Even before developing tools to facilitate the use of the modelling technique, (1) it should be determined if, and to what extent the technique and the resulting models can be integrated with task analysis and software engineering approaches. If such an integration is not possible, or only to a limited extent, then any other effort is useless. Otherwise, (2) an integration can be established, which is presumably not an easy undertaking. After the backbone of an overall design approach is thus created, (3) it may be refined, completed, and complemented with the required tools. Whereas the former two steps may take place as an entirely theoretical project, for the success of this last step practical experience will be inevitable, because only then the weak points and the gaps of the approach will show up. 
(c) The present approaches to formal modelling have almost exclusively been used within the research domain of Cognitive Ergonomics. This has led to a situation where design has commonly be exemplified by small-scale studies in which either students, or even the authors, of a certain design method took part as designers of a computer system with maybe ten different functions to perform some artificial task. Although there are exceptions, the point will be clear: in order to bridge the gap between theory and practice of interface design, the application of formal models in actual interface design is required in order to gain new theoretical insight. Only by means of full scale design examples will it be possible to show to the design community the advantages of using formal models. In this respect, one successful computer system is more valuable than ten research papers on interface design. Both the development of formal modelling techniques and the development of methods to design usable computer systems are best served by practical experience.

\section{References}

Arnold, A.G. and R.A. Roe, 1987. 'User errors in human-computer interaction'. In: M. Frese, E. Ulich and W. Dzida (eds.), Psychological issues of human computer interaction in the work place. Amsterdam: North-Holland.

Backus, J.W., F.L. Bauer, J. Green, C. Katz, J. McCarthy, P. Naur, A.J. Perlis, H. Rutishauer, K. Samelson, B. Vauquois, J.H. Wegstein, A. van Wijngaarden and M. Woodger, 1964. Revised report on the algorithmic language Algol 60. A/S regnecentralen, Copenhagen.

Barnard, P. and M. Harrison, 1989. 'Integrating cognitive and system models in human computer interaction'. In: A. Sutcliffe and L. Macaulay (eds.), Proc. People and computers V. Cambridge: Cambridge University Press. pp. 87-103.

Briggs. P., 1990. Do they know what they're doing? An evaluation of word-processor users' implicit and explicit task-relevant knowledge, and its role in self-directed learning. International Journal of Man-Machine Studies 32, 385-398.

Card, S.K., T.P. Moran and A. Newell, 1983. The psychology of human-computer interaction, Hillsdale, NJ: Erlbaum.

Diaper, D., 1989. 'Bridging the gulf between requirements and design'. In: Proc. Simulation in the development of user-interfaces. Brighton, 18-19 May. pp. 129-145.

Foltz, P.W., S.E. Davies and P.G. Polson, 1988. 'Transfer between menu systems'. In: E. Soloway, D. Frye and S.B. Sheppard (eds.), Proceedings CHI '88. Amsterdam: North-Holland. pp. 107-112.

Frohlich, D.M. and P. Luff, 1989. Some lessons from an exercise in specification. Human Computer Interaction 4, 101-123.

Gould, J.D. and C. Lewis, 1985. Designing for usability: key principles and what designers think. Communications of the ACM 28, 300-311. 
Green, T.R.G., 1990. 'Limited theories as a framework for human-computer interaction'. In: D. Ackerman, and M.J. Tauber (eds.), Mental models and human-computer interaction, Vol. 1. Amsterdam: North-Holland. pp. 3-39.

Green, T.R.G., F. Schiele and S.J. Payne, 1988. 'Formalisable models of user knowledge in human-computer interaction'. In: G.C. van der Veer, T.R.G. Green, J.M. Hoc and D.M. Murray (eds.), Working with computers: Theory versus outcome. London: Academic Press. pp. 3-46.

Innocent, P.R., M.J. Tauher, G.C. van der Veer, S. Guest, F.G. Haselager, F.G. McDaid, I. Oestreicher and Y. Waern, 1988. 'Representation of the user interface. Comparison of descriptions of interfaces from a designers point of view'. In: R. Speth (ed.), Research into networks and distributed applications. Amsterdam: North-Holland. pp. 345-359.

Jackendoff, R., 1983. Semantics and cognition. Cambridge, MA: MIT Press.

Karat, J., 1988. 'Software evaluation methodologies'. In: M. Helander (eds.), Handbook of human-computer interaction. Amsterdam: North-Holland. pp. 891-903.

Kieras, D., 1988. 'Towards a practical GOMS model methodology for user interface design'. In: M. Helander (ed.), Handbook of human-computer interaction. Amsterdam: North-Holland.

Kieras, D. and P.G. Polson, 1985. An approach to the formal analysis of user complexity. International Journal of Man-Machine Studies 22, 365-394.

Knowles, C., 1988. 'Can cognitive complexity theory (CCT) produce an adequate measure of system usability?' In: D.M. Jones and R. Winder (eds.), Proc. People and computers IV. pp. 291-307. Cambridge: University Press. Cambridge.

Lerch, F.J., M.M. Mantei and J.R. Olson, 1989. 'Skilled financial planning: The cost of translating ideas into action'. In: Proc. CHI '89: Wings for the mind. New York: ACM. pp. 121-126.

Moran, T.P., 1981. The command language grammar: A representation for the user-interface of interactive systems. International Journal of Man-Machine Studies 15, 3-50.

Moran, T.P., 1983. 'Getting into the system: External-internal task mapping analysis'. In: A. Janda (ed.), Proc. CHI '83. New York: ACM. pp. 45-49.

Murray, D.M., 1988. A survey of user cognitive modelling. National Physical Laboratory, NPL Report DITC 92/87.

Nielsen, J., 1986. A virtual protocol for computer-human interaction. International Journal of Man-Machine Studies 24, 301-312.

Nielsen, J., 1990. A meta-model for interacting with computers. Interacting with Computers 2, 137-160.

Norman, D.A., 1983. 'Some observations on mental models'. In: D. Gentner and A.L. Stevens (eds.), Mental models. Hillsdale, NJ: Erlbaum, pp. 7-14.

Oberquelle, H., 1983. 'On models and modelling in human-computer co-operation'. In: G.C. van der Veer, M.J. Tauber, T.R.G. Green and P. Gorny (eds.), Readings on cognitive ergonomics Mind and computers. Berlin: Springer-Verlag. pp. 26-43.

Payne, S.J., 1984. 'Task action grammar'. In: B. Shackel (ed.), Proc. Interact '84. Amsterdam: North-Holland. pp. 139-144.

Payne, S.J., 1987. 'Complex problem spaces: Modelling the knowledge needed to use interactive devices'. In: H.J. Bullinger, B. Shackel (eds.), Proc. Interact '87. Amsterdam: North-Holland. pp. 203-208.

Payne, S.J. and T.R.G. Green, 1983. 'The user's perception of the interaction language: A two-level model'. In: Proc. CHI '83: Human Factors in Computing Systems. New York: ACM. pp. 202-206.

Payne, S.J. and T.R.G. Green, 1986. Task-action grammars: A model of the mental representations of task languages. Human-Computer Interaction 2, 93-133.

Payne, S.J. and T.R.G. Green, 1989. The structure of command languages: An experiment on task-action grammar. International Journal of Man-Machine Studies 30, 213-234. 
Polson, P.G., 1987. 'A quantitative theory of human-computer interaction'. In: J.M. Carroll (ed.), Interfacing thought: Cognitive aspects of human-computer interaction. Cambridge, MA: MIT Press. pp. 184-235.

Polson, P.G., 1988. 'The consequences of consistent and inconsistent user interfaces'. In: R. Guindon (ed.), Cognitive science and its applications for human-computer interaction. Hillsdale, NJ: Erlbaum. pp. 59-108.

Polson, P.G. and D.E. Kieras, 1985. 'A quantitative model of the learning and performance of text editing knowledge'. In: L. Borman and B. Curtis (eds.), Proc. CHI '85. New York: ACM. pp. 207-212.

Preece, J. and L. Keller (eds.), 1990. Human-computer interaction: Selected readings. London: Prentice-Hall.

Rassmussen, J., 1987. 'Cognitive engineering'. In: H.J. Bullinger and B. Shackel (eds.), Proc. Interact '87. Amsterdam: North-Holland. pp. xxv-xxx.

Reisner, P., 1981. Formal grammar and human factors design of an interactive graphics system. IEEE Transactions on Software Engineering 7, 229-240.

Reisner, P., 1983. 'Analytic tools for human factors of software'. In: A. Blaser, M. Zoeppritz (eds.), Proc. Enduser systems and their human factors. Lecture Notes in Computer Systems no. 150. Berlin: Springer-Verlag, pp. 94-121.

Reisner, P., 1984. 'Formal grammar as a tool for analyzing ease of use: Some fundamental concepts'. In: J.C. Thomas and M.L. Schneider (eds.), Human factors in computer systems. Norwood, NJ: Ablex. pp. 53-78.

Richards, J.N.J., H.E. Bez, D.T. Gittins and D.J. Cooke, 1986. On methods for interface specification and design. International Journal of Man-Machine Studies 24, 545-568.

Roberts, T.L. and T.P. Moran, 1984. The evaluation of text editors: Methodology and empirical results. Communications of the ACM 26, 265-283.

Roe, R., 1988. 'Acting systems design - An action theoretical approach to the design of man-computer systems'. In: V. de Keyser, T. Qvale, B. Wilpert and S.A. Ruiz Quintanilla (eds.), The meaning of work and technological options. New York: Wiley.

Rohr, G. and M.J. Tauber, 1984. 'Representational frameworks and models for human-computer interfaces'. In: G.C. van der Veer, M.J. Tauber, T.R.G. Green and P. Gorny (eds.), Readings on cognitive ergonomics - Mind and computers. Berlin: Springer-Verlag. pp. 8-25.

Sharratt, B.D., 1987. 'Top-down interactive systems design: Some lessons learnt from using command language grammar'. In: H.J. Bullinger and B. Schackel (eds.), Proc. Interact '87. Amsterdam: North-Holland. pp. 395-399.

Shneiderman, B., 1982. Multiparty grammars and related features for defining interactive systems. IEEE Transactions on Systems. Man and Cybernetics SMC-12, 93-133.

Simon, T., 1988. 'Analysing the scope of cognitive models in human-computer interaction: A trade-off approach'. In: D.M. Jones and R. Winder (eds.), Proc. People and computers IV. Cambridge: Cambridge University Press. pp. 79-93.

Smith, S.L. and J.N. Mosier, 1984. Design guidelines for user-system interface software. Bedford, MA: The Mitre Corporation.

Summersgill, R, and D.P. Browne, 1989. Human factors: Its place in system development methods. Sigsoft 14, 227-234.

Tauber, M.J., 1988. 'On mental models and the user interface'. In: G.C. van der Veer, T.R.G. Green, J.M. Hoc and D.M. Murray (eds.), Working with computers: Theory versus outcome. London: Academic Press. pp. 89-119.

Tauber, M.J., 1990. ETAG: Extended Task Action Grammar - A language for the description of the user's task language'. In: D. Diaper, D. Gilmore, G. Cockton and B. Shackel (eds.), Proc. Interact '90. Amsterdam: North-Holland. pp. 163-168.

Van der Veer, G.C., 1989. Visual aspects of user interfaces - A psychological view. Notizen zu Interaktiven Systemen 17, 19-27. 
Van der Veer, G.C., 1990. Human-computer interaction: Learning, individual differences, and design recommendations. Ph.D. Thesis, Free University, Amsterdam.

Van der Veer, G.C., F. Yap, D. Broos, K. Donau and M.J. Fokke, 1990. 'ETAG - Some applications of a formal representation of the user interface'. In: D. Diaper et al. (eds.), INTERACT ' 90 - Third IFIP Conference on Human-Computer Interaction. Amsterdam: North-Holland.

Vossen, P.H., S. Sitter and J.E. Ziegler, 1987. 'An empirical validation of cognitive complexity theory'. In: H.J. Bullinger and B. Shackel (eds.), Proc. Interact '87. Amsterdam: North-Holland. pp. 203-208.

Walsh, P., 1989. 'Analysis for Task Object Modelling (ATOM): Towards a method of integrating task analysis with Jackson System development for user interface software design'. In: D. Diaper (ed.), Task analysis for human-computer interaction. Chichester: Ellis Horwood.

Whitefield, A., 1987. 'Models in human computer interaction: A classification with special reference to their uses in design'. In: H.J. Bullinger and B. Shackel (eds.), Proc. Interact '87. Amsterdam: North-Holland. pp. 57-63.

Wilson, M.D., P.J. Barnard, T.R.G. Green and A. MacLean, 1988. 'Knowledge based task analysis for human-computer systems'. In: G.C. van der Veer, T.R.G. Green, J.M. Hoc and D.M. Murray (eds.), Working with computers: Theory versus outcome. London: Academic Press. pp. 47-87.

Van Wijngaarden, A., B.J. Mailloux, J.E.L. Peck and C.H.A. Koster, 1969. Report on the algorithmic language ALGOL 68. Numer. Math. 14. 79-218.

Young, R.M., T.R.G. Green and T. Simon, 1989. 'Programmable user models for predictive evaluation of interface designs'. In: K. Bice and C. Lewis (eds.), Proc. CHI '89. New York: ACM. pp. 15-19. 